\title{
PROJETO I HEART ENGENHARIA: MELHORIA DA GRADUAÇÃO COMO COMBATE À EVASÃO UNIVERSITÁRIA
}

\author{
Marco Aurelio Nespolo Vomstein - marco.vomstein@gmail.com \\ Bruna Messias Dourado - brunadourado110@gmail.com \\ Diovana Fátima Menegat da Rocha - diovanafmr@gmail.com \\ Elisa De Oliveira - elisa_papes@hotmail.com \\ Gabriel Günther Da Rosa - gabrielgunther1@ hotmail.com \\ Universidade do Estado de Santa Catarina \\ R. Paulo Malschitzki, 200 - Zona Industrial Norte \\ 89219-710 - Joinville - SC
}

Resumo: Este trabalho descreve a atuação do projeto de ensino I Heart Engenharia, do Programa de Educação Tutorial - PET Engenharia Elétrica da Universidade do Estado de Santa Catarina - Udesc Joinville. É dado foco a três atividades principais: o Laboratório Cooperativo (LabCoop) que consiste em um ambiente aberto à comunidade acadêmica com equipamentos e componentes para execução de atividades relacionadas à graduação e extraclasse; o projeto Ciclo de Treinamentos $(C d T)$ que realiza minicursos, palestras e outras atividades de capacitação para a graduação e a comunidade externa; e ações relacionadas ao acolhimento dos ingressantes no curso de Engenharia Elétrica, em especial a atividade Calouro Voluntário, que permite ao ingressante um contato com a tríade universitária por meio da participação em atividades do PET. Como forma de mensurar o impacto desta última ação, foi feita uma análise das respostas de um formulário aplicado aos participantes do Calouro Voluntário que demonstrou, entre outros resultados, ser efetiva para a inserção dos atingidos em projetos dentro da universidade. Ainda, a partir do cumprimento dos objetivos traçados nos editais internos à universidade que os projetos LabCoop e CdT estão submetidos, percebe-se a promoção da capacitação dos estudantes e de um ambiente adequado para a realização de atividades práticas. Os fatores apresentados contribuem para o objetivo principal do projeto de diminuição da evasão na graduação, em especial nas fases iniciais do curso de engenharia elétrica.

Palavras-chave: Evasão universitária. Melhoria da graduação. Engenharia Elétrica. Projeto de ensino.

\section{INTRODUÇÃO}

Pode-se definir projeto de ensino como toda atividade que tem por objetivo a melhoria do processo de ensino-aprendizagem, suprindo as sequelas deixadas em sala de aula. Entende-se que os projetos de ensino são necessários para complementar a formação dos estudantes universitários e servem como auxílio a problemas que estão atrelados a uma sociedade desigual e excludente.

Tendo em vista, por exemplo, a grande demanda de calouros nas universidades públicas que se deslocam de diversas regiões do país e proveem de escolas públicas e privadas, constata-se uma elevada disparidade na bagagem de conteúdos que esses trazem do ensino médio. 
Cury (2002) define que para analisar a situação do ensino no Brasil não deve-se excluir a relação entre a educação e a situação econômica do país, confirmando assim que a renda das famílias está diretamente ligada ao acesso e dedicação dos estudantes na escola.

Outro problema comum nos cursos de engenharia nas universidades públicas é a evasão nos primeiros semestres, durante o ciclo básico do curso. Essa evasão é causada principalmente pelo grande número de reprovações e pela dificuldade em adaptar-se à rotina acadêmica, muitas vezes em conjunto com uma ocupação profissional e a vida social.

Ferreira et. al. (2001) afirma que uma das causas da evasão é a dificuldade dos ingressantes em se adaptarem à nova rotina imposta pela universidade, que configura um ambiente muito mais exigente que o ensino médio. Além disso, de acordo com Bardagi e Hutz (2009) a problemática da evasão é atribuída diretamente a problemas individuais dos alunos, mas deve ser vista tendo origem na instituição como um todo.

Dessa forma, os projetos de ensino incitam que os alunos considerem-se como responsáveis pela construção do seu próprio conhecimento, estimulando a curiosidade acerca do conteúdo de forma natural e proporcionando práticas de ensino que vão além das aulas expositivas. Logo, a finalidade dos projetos de ensino vai além do auxílio aos estudantes visto que servem de amparo aos professores e à universidade, provocando uma avaliação crítica em todos os envolvidos.

Com esse enfoque, o projeto I Heart Engenharia foi desenvolvido pelo Programa de Educação Tutorial (PET) Engenharia Elétrica, com o propósito de sanar as deficiências da graduação em Engenharia Elétrica da Universidade do Estado de Santa Catarina - Centro de Ciências Tecnológicas (Udesc-CCT). A fim de trabalhar diretamente nas problemáticas anteriormente citadas, o projeto atua diretamente nas demandas dos professores, alunos e colaboradores. O I Heart Engenharia tem por objetivo melhorar a condição de ensino de engenharia da Udesc-CCT através de ações variadas, além de motivar os graduandos e recémingressados no curso de Engenharia Elétrica.

\section{HISTÓRICO DO PROJETO}

A lei de 11.180/2005 instituiu oficialmente o Programa de Educação Tutorial (PET) com o propósito de aumentar a qualidade do ensino superior através da indissociabilidade da tríade universitária: ensino, pesquisa e extensão.

O PET Engenharia Elétrica da Udesc foi criado em 1994 e possui como missão o fortalecimento do ensino superior, do desenvolvimento tecnocientífico e da sociedade de maneira indissociável, com base nas habilidades de engenharia e educação tutorial. No grupo, são realizadas atividades da tríade universitária com uma organização interna de projetos de ensino, pesquisa e extensão.

No PET Engenharia Elétrica inicialmente eram realizadas algumas atividades de ensino contínuas, porém essas não estavam vinculadas a nenhum projeto. Tais atividades eram, por exemplo, melhorias de disciplinas curriculares, desenvolvimento de protótipos, gestão do Laboratório Cooperativo (LabCoop), e atividades diversificadas com os calouros do curso de Engenharia Elétrica. Dessa forma, constatou-se a necessidade de criar um projeto para englobar tais atividades, surgindo assim o I Heart Engenharia, com objetivo principal sanar as lacunas no ensino da Engenharia Elétrica e do Centro de Ciências Tecnológicas.

Atualmente, o projeto I Heart Engenharia manteve as atividades relacionadas ao auxílio ao ensino e acrescentou outras, de acordo com a necessidade dos docentes e discentes do curso. Entre elas há o projeto Calouro Voluntário que visa inserir os calouros do curso de Engenharia Elétrica nos projetos de ensino, pesquisa e extensão da Udesc, buscando dessa forma auxiliar no combate à evasão universitária, uma vez que os projetos promovem a 
integração do calouro no novo ambiente acadêmico. Além disso, são oferecidos minicursos, palestras e oficinas, totalmente gratuitos dentro da universidade, promovidas pelo projeto Ciclo de Treinamentos (CdT) que corrobora o desenvolvimento técnico dos estudantes. Já o Laboratório Cooperativo (LabCoop), também inserido no projeto I Heart Engenharia, consiste em um espaço de uso livre para os estudantes com computadores, osciloscópios, fontes e demais equipamentos necessários para que os alunos possam realizar os trabalhos oriundos das disciplinas e pesquisas, e também para realizar atividades extracurriculares.

\section{ESTRUTURA E METODOLOGIAS}

Para poder entender melhor o que desempenha o projeto, faz-se necessária uma divisão entre a sua estrutura e uma apresentação das atividades atualmente desenvolvidas, que terão seus resultados apresentados no decorrer deste artigo.

\subsection{Estrutura do projeto}

O I Heart Engenharia compreende a uma das iniciativas voltadas para o ensino dentro do PET e, por conta disto, está submetido a uma série de procedimentos padrões do Programa. A participação dentro do I Heart é semestral e seu ingresso ocorre durante o período de planejamento do grupo, onde os discentes integrantes do PET que estiverem interessados em participar do projeto indicam o seu interesse, sendo um destes o coordenador, responsável por acompanhar e designar as atividades, mas tão responsável por elas quanto os demais membros.

Para a parte organizacional, o I Heart Engenharia utiliza diversas ferramentas e metodologias. Uma delas é o $3 \mathrm{G}$, ou 3 Gerações, documento padrão do PET Engenharia Elétrica da Udesc que é utilizado para registrar as atividades realizadas no passado, acompanhar as atividades do presente e dar sugestões de realizações futuras. Também é utilizado um acompanhamento dos membros, no qual estes registram suas atividades num período quinzenal, buscando auxiliar e equilibrar o número de atividades e sua complexidade dentre todos os integrantes do projeto.

Com o auxílio dos 3Gs, no início de cada semestre o projeto tem um período de planejamento próprio, onde verifica as atividades que se propõe a realizar durante o semestre letivo, visando atingir as metas determinadas pelo PET no decorrer do ano. As atividades são planejadas em conformidade com o planejamento estratégico do PET que estabelece metas a serem batidas durante um tempo, normalmente de 2 anos, a fim de medir os resultados dos seus projetos, incluindo o I Heart Engenharia.

Após as transformações que sofreu ao longo dos anos, o projeto conta com diversas iniciativas e, também, continua com projetos coordenados internamente: o LabCoop e o CdT. Estes dois projetos, sendo um de ensino e um de extensão, são submetidos aos editais da Udesc, o Prapeg (Programa de Apoio ao Ensino de Graduação) e o Paex (Programa de Apoio à Extensão Universitária), respectivamente. Estes editais garantem aos projetos um fomento, que auxilia na compra de equipamentos, deslocamentos, dentre outras possibilidades, além de também servirem como parâmetros para avaliar os resultados alcançados pelo projeto.

\subsection{Atividades e metodologias}

De modo geral, as ações do I Heart Engenharia têm foco em 3 atividades: o LabCoop, o CdT e atividades de acolhimento dos ingressantes no curso. Ainda, podem ser citadas atividades gerais de melhorias de disciplinas e laboratórios que são desenvolvidas a partir da verificação da demanda. 
O LabCoop surge a partir da demanda por utilização de espaço físico adequado para o desenvolvimento de trabalhos e atividades relacionados com a Engenharia Elétrica e áreas afins em horário extraclasse. A partir disso, o LabCoop tem o intuito de estimular o interesse dos graduandos pelo curso e fomentar o desenvolvimento tecnológico e a criatividade. $\mathrm{O}$ Laboratório fornece aos discentes voluntários e bolsistas envolvidos a oportunidade de desenvolver protótipos para aplicação na instituição e na sociedade, a partir de demanda avaliada. Estes discentes participantes, junto a membros do I Heart Engenharia, desenvolveram uma estrutura interna já bem consolidada, com a departamentalização das atividades recorrentes do projeto.

O CdT é um projeto de ensino e extensão que tem como principal intuito a reprodução de conhecimento dos acadêmicos para os demais estudantes, buscando atingir tanto pessoas que ainda não tiveram acesso ao mesmo, bem como reforçar esses conceitos àqueles que já têm certa familiaridade com o assunto. Tais atividades são realizadas por meio de minicursos, palestras, rodas de conversa e visitas técnicas, sendo esses oferecidos aos graduandos não somente da engenharia elétrica, mas, também, para os dos outros cursos existentes no campus. Além disso, essas atividades contemplam conteúdos diversos e multidisciplinares. Para implementar tais atividades, o CdT trabalha com o efeito multiplicador, priorizando sempre a troca de conhecimentos em ciclo, de forma a incentivar que aqueles que estão agora aprendendo sobre os assuntos ofertados nas palestras e minicursos, repassem os conhecimentos adquiridos adiante.

Tema de grande importância para o PET Engenharia Elétrica, o acolhimento dos calouros é a atividade com maior atuação do I Heart Engenharia, com a realização de diversos eventos e iniciativas que buscam trazer aos ingressantes um ambiente ainda melhor para o início do seu curso. Nesta ideia, o I Heart Engenharia vai à sala de aula, na disciplina de Introdução à Engenharia Elétrica, realizar uma apresentação completa e detalhada sobre o PET e seu histórico, bem como apresenta sua organização atual e projetos envolvidos. Junto a esta aula, lhes é apresentada outra iniciativa do projeto: o Calouro Voluntário. No Calouro Voluntário, os alunos interessados são inscritos para participarem, durante o seu primeiro semestre na universidade, das atividades realizadas pelo PET, como conhecendo a pesquisa de alguns dos participantes, as estruturas organizacionais, e os demais projetos relacionados ao Programa. A participação nesta iniciativa traz a eles maior conhecimento sobre o curso e contato com diversos projetos que possam vir a participar.

Ainda relacionado ao acolhimento dos calouros, algumas outras atividades também merecem destaque. O Papo com as Calouras é uma iniciativa recente do projeto que consiste em uma roda de conversa das ingressantes no curso com diversas mulheres ligadas à Universidade, com relatos de experiências, desafios encontrados e conselhos de como proceder em diversas situações de machismo. No Tour com os Calouros, os ingressantes no curso são levados para conhecer as instalações e laboratórios do Departamento de Engenharia Elétrica (DEE).

\section{RESULTADOS E DISCUSSÃO}

Considerando as 3 principais atividades do I Heart Engenharia apresentadas, podemos analisar os resultados alcançados a partir de parâmetros de planejamento distintos. Para o LabCoop será utilizado como parâmetro os objetivos estabelecidos no último edital Prapeg submetido pelo projeto. Em relação ao CdT, os resultados serão analisados a partir do que foi proposto no edital Paex. Por último, em relação às atividades de acolhimento dos ingressantes no curso, serão analisadas as respostas de uma pesquisa aplicada por meio de formulário 
online que contou com dados de 27 participantes da atividade entre os semestres de 2015/1 e $2019 / 2$

Durante os anos de 2018 e 2019, o projeto LabCoop realizou todas as atividades previstas no edital Prapeg, que teve vigência neste período. Dentre as ações, podem ser citadas a elaboração de roteiros para a implementação de circuitos eletrônicos, a montagem de novos protótipos, a manutenção constante da infraestrutura do laboratório, a gestão de recursos e da logística de utilização do local pela graduação e a expansão da equipe do projeto que atualmente conta com 1 docente, 8 discentes voluntários e 2 discentes bolsistas. Com o registro de pessoas que utilizaram o laboratório durante o período, estima-se 1000 atingidos nos últimos dois anos. Como reconhecimento da importância do LabCoop para a graduação, o projeto ficou na primeira colocação no edital Prapeg atual, dentre cinco projetos vinculados ao Departamento de Engenharia Elétrica da Udesc.

A partir dos objetivos traçados no último edital Paex, o Ciclo de Treinamentos realizou minicursos, palestras e capacitação de formas diversas. As atividades realizadas pelo projeto possibilitaram a capacitação e a atualização dos alunos em relação às novas tecnologias, em especial em se tratando de softwares. Isso foi possível, na medida em que essas ações proporcionaram o aprimoramento dos ministrantes, como consequência do efeito multiplicador inserido no projeto. Propiciaram também o desenvolvimento de conhecimentos extracurriculares, que auxiliaram nas disciplinas, e aproximaram o público das ferramentas encontradas no mercado de trabalho. Até 2018 o CdT atuou na Semana Acadêmica de Engenharia Elétrica - Seteel e, a partir da criação da Semana de Eventos Integrados - SEI, em 2019, o projeto passou a atingir com maior eficiência todos os cursos do centro, por meio da organização de minicursos e oficinas gratuitas que atingiram cerca de 90 estudantes.

Com relação ao formulário aplicado aos participantes do Calouro Voluntário, na primeira pergunta que questiona os motivos que os levaram a ingressar na atividade podemos destacar a recorrência de algumas respostas: a oportunidade de conhecer e realizar atividades conjuntas com os projetos da universidade, descobrir as atividades e oportunidades que o ambiente acadêmico tem a oferecer, conhecer melhor o curso de engenharia elétrica e suas áreas, aproximar-se das atividades realizadas pelo grupo PET, entender os conceitos da tríade universitária e realizar atividades práticas na primeira fase.

A segunda pergunta aborda aspectos que o projeto oportunizou como melhoria da formação. Para esse questionamento foram apresentadas 4 opções para serem assinaladas e um espaço para inserir outros aspectos não mencionados. Como resultado, $44 \%$ dos respondentes relataram que tiveram características de trabalho em equipe aprimoradas; $56 \%$ indicaram um aumento do conhecimento técnico; $70 \%$ assinalaram que tiveram um aumento no contato com o público externo e $93 \%$ relataram o aumento do contato com outros projetos da universidade. Ainda houve respostas indicando a participação em debates, aumento do contato com a docência, desenvolvimento da habilidade de resolver problemas, conhecimento de áreas de pesquisa e o despertar da curiosidade.

A terceira pergunta questionou o aumento do interesse pela engenharia elétrica após a experiência como Calouro Voluntário, com escala de 1 (nenhum aumento) a 5 (aumento muito grande). Os resultados desta pergunta são apresentados na Figura 1 na qual se pode ver que $74 \%$ foram nas opções 4 e 5 , o que, de acordo com a escala utilizada, demonstraram um aumento considerável do interesse. 
Figura 1 - Respostas dos ingressantes no curso sobre o aumento do interesse pela engenharia elétrica após a experiência como Calouro Voluntário.

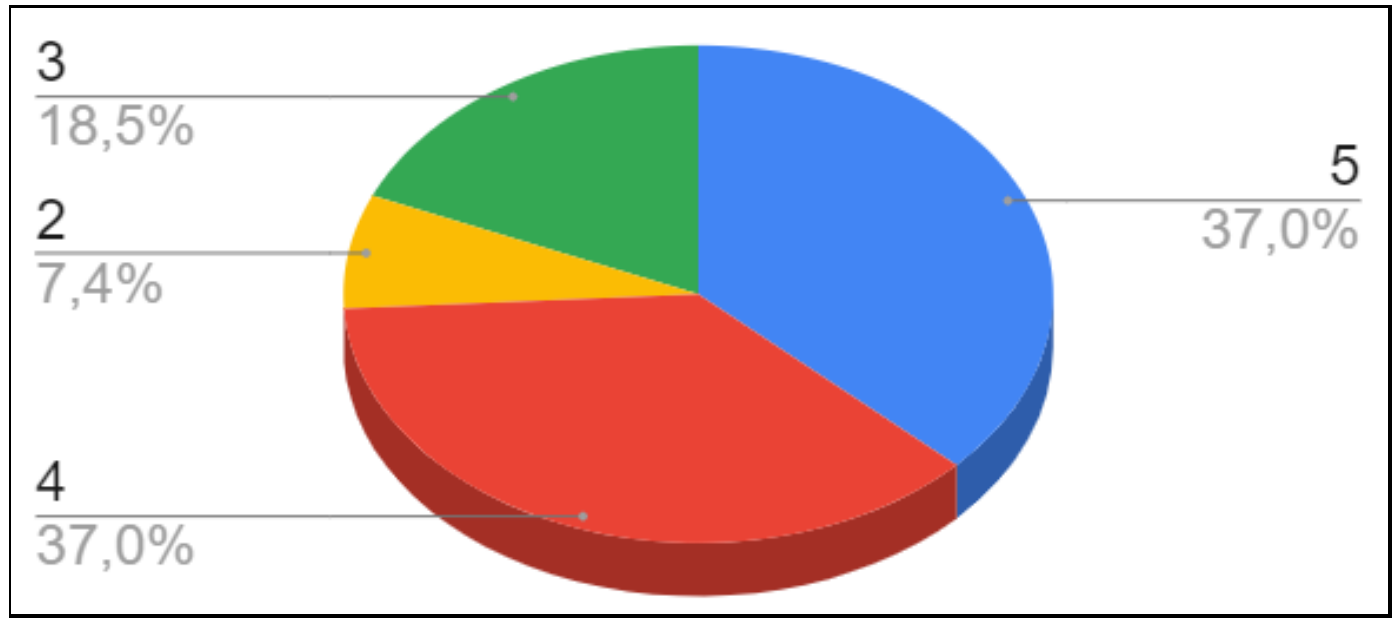

Fonte: Dos autores.

O último questionamento foi sobre o ingresso do estudante em projetos da universidade e o quanto a experiência como Calouro Voluntário influenciou nesta decisão. Novamente com escala de 1 (nenhuma influência) a 5 (muita influência). Das respostas, constata-se que $2 / 3$ dos participantes ingressaram em algum projeto da universidade. Destes, 83,3\% relataram um grau de influência entre 4 e 5 do Calouro Voluntário. Ainda, dentre os que não entraram em algum projeto, $44,4 \%$ comentaram que a experiência no projeto os incentivaram a realizar processos seletivos.

Por meio destas respostas, podemos constatar a eficiência do Calouro Voluntário como forma de aumentar o interesse pelo curso e incentivo à participação em projetos.

Outras atividades com os ingressantes no curso como o Tour com os Calouros ajudam a promover um ambiente universitário acolhedor. Em especial o Papo com as Calouras que, por apresentar relatos e experiências de professoras e alunas da instituição, faz com que as ingressantes se sintam amparadas e apoiadas em um curso de maioria masculina.

\section{CONSIDERAÇÕES FINAIS}

O objetivo deste trabalho foi demonstrar a importância do projeto I Heart Engenharia, formulado a partir de iniciativa do grupo PET da instituição, para diminuir os índices de evasão do curso de Engenharia Elétrica da Udesc-CCT, tornar o ambiente acadêmico mais acolhedor e principalmente auxiliar no desenvolvimento do conhecimento dos acadêmicos com programas de auxílio ao ensino realizado em sala de aula. Dessa forma, através das análises aqui apresentadas pode-se constatar a importância do projeto, visto o maior engajamento dos alunos nas atividades que englobam a graduação.

A importância também é reconhecida pelo corpo discente do departamento de Engenharia Elétrica, visto que o LabCoop ficou com a primeira colocação no edital Prapeg, como já citado anteriormente. Além disso, o Laboratório impacta todo o centro, por ser o único ambiente de livre acesso aos alunos com o espaço e os equipamentos necessários, para que esses possam desenvolver suas suas atividades extracurriculares e de pesquisa.

O programa CdT é parte fundamental na semana acadêmica e no desenvolvimento dos alunos. Visto que, nele há o repasse do conhecimento de aluno para aluno, de forma gratuita e 
relevante, abrangendo minicursos e palestras dos mais variados temas e assuntos, além de visitas-técnicas.

O projeto também auxilia na difusão do conhecimento da tríade universitária, seja através das aulas de IEE ou do Calouro Voluntário. Dessa forma, os acadêmicos têm a possibilidade de buscar uma formação completa através da indissociabilidade da tríade, por meio da ligação a projetos que têm filosofias semelhantes às do PET. A partir do formulário aplicado, concluise que o Calouro Voluntário auxilia no crescimento do número de alunos participando dos projetos na universidade, que agora passam a ser participantes ativos na execução da tríade interligada.

Outra atividade que vem tendo maior visibilidade dentro da instituição e sendo reproduzido por outros grupos é o Papo com as Calouras, que ocorre com o intuito de incentivar que as ingressantes tenham referências femininas no âmbito universitário, incentivando o apoio mútuo entre mulheres em um meio ainda tratado como masculino, que é a engenharia.

Em cursos com índices altos de reprovação e evasão nas fases iniciais, é essencial a atuação de projetos de ensino que permitem uma abordagem prática e proporcionem um ambiente acolhedor para contribuir com a permanência estudantil, ficando evidente, a partir das atividades desenvolvidas pelo I Heart Engenharia, o resultado de seu objetivo de combate à evasão e auxílio ao ensino na Udesc-CCT.

\section{Agradecimentos}

Agradecemos a Universidade do Estado de Santa Catarina pelo apoio institucional e financeiro dado ao Programa de Educação Tutorial para a realização das atividades, o FNDE pelos auxílios de custeio e bolsas dos integrantes do grupo e todos os integrantes envolvidos no desenvolvimento e execução das atividades do projeto I Heart Engenharia.

\section{REFERÊNCIAS}

BARDAGI, Marucia Patta; HUTZ, Cláudio Simon. "Não havia outra saída": percepções de alunos evadidos sobre o abandono do curso superior. Psico-USF (Impr.) [online]. 2009, vol.14, n.1, pp.95-105. ISSN 1413-8271.

https://doi.org/10.1590/S1413-82712009000100010.

CURY, Carlos Roberto Jamil. A Educação Básica no Brasil. Educ. Soc. [online]. 2002, vol.23, n.80, pp.168-200. ISSN 1678-4626. https://doi.org/10.1590/S0101$\underline{73302002008000010 .}$.

FERREIRA, Joaquim Armando; ALMEIDA, Leandro S. and SOARES, Ana Paula C. Adaptação académica em estudante do $1^{\circ}$ ano: diferenças de gênero, situação de estudante e curso. Psico-USF (Impr.) [online]. 2001, vol.6, n.1, pp.1-10.

ISSN 1413-8271. https://doi.org/10.1590/S1413-82712001000100002. 


\title{
Evento On-line
}

\section{HEART ENGINEERING PROJECT: IMPROVING GRADUATION AS A FIGHT AGAINST UNIVERSITY EVASION}

\begin{abstract}
This document describes the work of the teaching project I Heart Engenharia, of the Tutorial Education Program - PET Electrical Engineering from the Santa Catarina State University - Udesc Joinville. Focus is given to three main activities: the Cooperative Laboratory (LabCoop) which consists of an environment open to the academic community with equipment and components for carrying out activities related to graduation and extraclass; the Training Cycle (CdT) project, which offers short courses, lectures and other training activities for undergraduate students and the external community; and actions related to welcoming new students to the Electrical Engineering course, especially the Voluntary Freshman activity, which allows new students to have contact with the university triad through participation in PET activities. As a way of measuring the impact of this last action, an analysis of the responses of a form applied to the participants of the Voluntary Freshman was made, which demonstrated, among other results, to be effective for the insertion of those affected in projects within the university. Still, from the fulfillment of the objectives outlined in the public notices to the university that the LabCoop and CdT projects are submitted to, it is noticed the promotion of the training of students and an adequate environment for carrying out practical activities. The factors presented contribute to the main objective of the project to reduce dropout in graduation, especially in the initial phases of the electrical engineering course.
\end{abstract}

Keywords: university evasion, graduation improvement, electrical engineering, teaching project. 